\title{
Les gènes humains de la réparation de I'ADN
}

Le matériel génétique de toute cellule, soumis à l'action délétère de nombreux agents génotoxiques endogènes ou exogènes, doit cependant être répliqué et transmis avec une extraordinaire fidélité. Les mécanismes de réparation qui assurent cette stabilité ont commencé à être appréhendés chez l'homme grâce à l'étude de plusieurs maladies associées à des hypersensibilités à ces agents génotoxiques, telles que le Xeroderma pigmentosum, le syndrome de Cockayne ou la trichothiodystrophie. Huit gènes de réparation de l'ADN ont ainsi été clonés et on a montré qu'ils étaient mutés dans l'une ou l'autre de ces maladies. Ces gènes humains ont des analogues chez la bactérie, la levure ou la drosophile et sont souvent indispensables à la vie cellulaire. L'étude des produits de ces gènes permet naturellement de mieux comprendre les mécanismes fondamentaux de la réparation, mais aussi de la transcription de l'ARN à laquelle certaines enzymes de réparation collaborent. En effet, au moins deux gènes de réparation codent pour des protéines qui possèdent une activité hélicase ADN/ARN utilisée pour l'initiation de la transcription. Enfin, les mécanismes de réparation de l'ADN jouent un rôle fondamental dans l'intégrité des cellules nerveuses et dans l'initiation de la cancérogenèse chez l'homme.

'intégrité du matériel génétique contenu dans toute cellule vivante est continuellement remise en cause par une variété d'agents génotoxiques d'origine exogène (rayonnements ultraviolets ou ionisants, cancérogènes chimiques, médicaments antitumoraux) ou endogène (instabilité chimique des bases nucléiques, radicaux libres produits au cours du métabolisme énergétique cellulaire). De nombreux systèmes de réparation protègent les cellules contre l'accumulation délétère de lésions sur leur
ADN, par des voies biochimiques assez bien conservées des bactéries à l'homme. Le système de réparation par excision-resynthèse de nucléotides est le plus important et le plus efficace pour éliminer la grande majorité des lésions de l'ADN. Les lésions produites, par les rayons ultraviolets (les deux lésions majeures affectent deux pyrimidines adjacentes: les dimères de pyrimidines et les pyrimidines (6-4) pyrimidones [1]), par la plupart des cancérigènes chimiques (aflatoxine $B_{1}$, hydrocarbures polycycliques, amines aromatiques), ou par certain 


\section{RÉEÉRENCES}

1. Sarasin A, Renault G, BlanchetBardon C, Boue J, Dumez Y. Le Xeroderma pigmentosum: caractéristiques cliniques génétiques et cellulaires. méde cine/sciences $1988 ; 4: 608-17$.

2. Sarasin A, Moustacchi E. Maladies génétiques associées à une hypersensibilité cellulaire à des agents génotoxiques et à une forte prédisposition aux cancers. In : Dubertret L., ed. Encyclopédie des Cancers. Paris: Flammarion, 1992: 16-40.

3. Hanawalt PC, Sarasin A. Cancer prone hereditary diseases with DNA processing abnormalities. Trends Cenet 1986; 2 : 124-9.

4. Cleaver JE. Defective repair replication of DNA in Xeroderma pigmentosum. Nature 1968; 218: 652-6.

5. Kraemer KH, Lee MM, Scotto J. Xeroderma pigmentosum. Arch Dermatol 1987 ; 123: 241-50.

6. I ehmann AR. Cockayne's syndrome and trichothiodystrophy: defective repair without cancer. Cancer Res 1987; 7 : 82-103.

7. Sarasin A. The paradox of DNA repairdeficient diseases. Cancer J 1991; 4 : 233-7.

8. Venema J, Mullenders LHF, Natarajan AT, van Zeeland AA, Mayne LV. The genetic effect in Cockayne syndrome is associated with a defect in repair of UV-induced DNA damage in transcriptionally active DNA. Proc Natl Acad Sci USA 1990; 87 : 4707-11.

9. Mellon I, Spivak G, Hanawalt PC. Selective removal of transcription-blocking DNA damage from the transcribed strand of the mammalian DHFR gene. Cell 1987; 51: 241-9.

10. Sarasin A, Robert-Knebelmann C, Stary A. La réparation de l'ADN et les réparatoses. Pathol Biol 1992; 272 : 101-10.

11. Venema J, van Hoffen A, Natarajan $A T$, van Zeeland AA, Mullenders A. The residual repair capacity of Xeroderma pigmentosum complementation group C fibroblasts is highly specific for transcriptionally active DNA. Nucleic Acids Res 1990 ;

ques), ou par certains médicaments (mitomycine $\mathrm{C}$, cis-platine, psoralène ponté à l'ADN par les rayons ultraviolets $\mathrm{A}$ ), sont éliminées avec une très grande efficacité par le système de réparation par excision. Ces réparations jouent un rôle fondamental du fait que les lésions de l'ADN entraînent une désorganisation complète de l'activité cellulaire : blocage de la transcription des gènes actifs, blocage de la réplication de l'ADN ou synthèse translésionnelle conduisant inévitablement à l'induction de mutations ou de remaniements chromosomiques. Il apparaît donc évident que l'absence de réparation peut conduire à une modification irréversible de la régulation de la prolifération cellulaire et initier un mécanisme de cancérogenèse. Cette hypothèse est fortement confortée par l'existence de maladies de la réparation de l'ADN qui montrent souvent une corrélation étroite entre sensibilité à un agent génotoxique et forte incidence de cancers plus ou moins spécifiques $[2,3]$. Parmi ces maladies, le Xeroderma pigmentosum (XP) est l'exemple le plus probant d'une relation directe entre l'absence de réparation de lésions induites par les rayons ultraviolets et le développement précoce de tumeurs cutanées sur la peau exposée au soleil [1-5]. Les études récentes portant sur des lignées cellulaires isolées de ces malades hypersensibles aux rayons ultraviolets ont permis d'isoler les premiers gènes humains de la réparation de l'ADN. Les analyses préliminaires de ces gènes ont apporté de grandes surprises concernant leur rôle biologique, mais aussi leur implication possible dans le mécanisme d'induction de cancers et éventuellement dans la dégénérescence neuronale.

\section{Les maladies d'hypersensibilité aux rayons ultraviolets}

Trois des principales hypersensibilités aux rayons ultraviolets sont liées à des anomalies plus ou moins sévères dans l'efficacité du mécanisme de réparation de l'ADN par l'excision-resynthèse. Elles entraînent toutes des maladies rares, transmises sur le mode récessif et non liées au sexe (les enfants malades sont homozygotes, alors que leurs deux parents, normaux vis-à-vis des agents génotoxiques, sont hétérozygotes). Les grandes caractéristiques cliniques et biochimiques de ces différentes maladies sont indiquées dans le Tableau I.

\section{Le Xeroderma pigmentosum}

Le Xeroderma pigmentosum est la maladie la plus sévère et la mieux connue. Les malades atteints de Xeroderma pigmentosum ont une photosensibilité extrême aux rayons ultraviolets et sont caractérisés par une très forte incidence de cancers de la peau dans les zones exposées au soleil. Une fréquence de 1000 à 4000 fois supérieure à celle de la population normale est en effet observée pour les tumeurs cutanées chez les malades atteints de Xeroderma pigmentosum [1-5]. Dans les cas extrêmement graves, les tumeurs de la peau apparaissent en nombre important sur le visage, les yeux, les mains et les avant-bras, dès l'âge de 4-5 ans. La mort peut survenir vers 10-12 ans, non seulement du fait du trop grand nombre de tumeurs, mais aussi d'infections répétées, de cachexie, etc. Environ $20 \%$ à $30 \%$ des malades ont des anomalies dégénératives neurologiques importantes. Les symptômes sont toutefois assez variables d'un malade à l'autre, indiquant une hétérogénéité génétique qui apparaîtra au niveau cellulaire. Les cellules des malades atteints de Xeroderma pigmentosum sont particulièrement sensibles in vitro aux rayons ultraviolets et à divers agents génotoxiques du fait d'anomalies dans le système de réparation par excision. Ces malades dits "XP classiques" représentent $70 \%-75 \%$ de tous les malades atteints de Xeroderma pigmentosum. La fusion cellulaire entre cellules somatiques de deux XP classiques, permet d'isoler un hybride cellulaire éventuellement capable de réparer l'ADN endommagé par les rayons ultraviolets. Cette technique démontre l'hétérogénéité génétique au niveau cellulaire et permet de définir des groupes de complémentation. Sept groupes de complémentation (de $\mathrm{A}$ à $\mathrm{G}$ ) 


\begin{tabular}{|c|c|c|c|c|c|c|c|c|c|}
\hline \multirow{3}{*}{ Maladies } & \multicolumn{9}{|c|}{$\begin{array}{l}\text { CARACTÉRISTIQUES CLINIQUES ET GÉNÉTIQUES DES MALADES HYPERSENSIBLES } \\
\text { AUX RAYONS ULTRAVIOLETS }\end{array}$} \\
\hline & \multirow{2}{*}{$\begin{array}{l}\text { Fréquence } \\
\text { relative }\end{array}$} & \multirow{2}{*}{$\begin{array}{l}\text { Sensibilité } \\
\text { aux UV }\end{array}$} & \multicolumn{2}{|c|}{ Taux de réparation } & \multirow{2}{*}{$\begin{array}{l}\text { Cancers } \\
\text { de la } \\
\text { peau }\end{array}$} & \multirow{2}{*}{$\begin{array}{l}\text { Anomalies } \\
\text { neuro- } \\
\text { logiques }\end{array}$} & \multirow{2}{*}{$\begin{array}{l}\text { Anomalies } \\
\text { des } \\
\text { cheveux }\end{array}$} & \multirow{2}{*}{$\begin{array}{l}\text { Gènes } \\
\text { déficients }\end{array}$} & \multirow{2}{*}{ Particularités } \\
\hline & & & $\begin{array}{l}\text { ADN } \\
\text { total }\end{array}$ & $\begin{array}{c}\text { Gènes } \\
\text { transcrits }\end{array}$ & & & & & \\
\hline $\begin{array}{l}\text { Xeroderma } \\
\text { pigmentosum } \\
X P-A\end{array}$ & élevée & +++ & $<5 \%$ & $<5 \%$ & ++ & ++ & - & XPAC & \\
\hline$X P-B$ & rare & ++ & $<10 \%$ & $<10 \%$ & + & t+ & - & ERCC3 & Identique au CS groupe \\
\hline$X P-C$ & élevée & + & $<15 \%$ & $100 \%$ & ++ & - & - & XPCC & $\begin{array}{l}\text { Réparation normale des } \\
\text { gènes transcrits }\end{array}$ \\
\hline XP-D & moyenne & ++ & $20-40 \%$ & $20-40 \%$ & ++ & + & - & ERCC2 & $\begin{array}{l}\text { - Quelques malades } \\
\text { associent le CS } \\
\text { - même groupe de } \\
\text { complémentation que le } \\
\text { TTD-2 }\end{array}$ \\
\hline$X P-E$ & rare & $+1-$ & $<50 \%$ & $<50 \%$ & $+1-$ & - & - & $?$ & $\begin{array}{l}\text { Défaut dans une pro- } \\
\text { téine qui se lie à l'ADN } \\
\text { endommagé }\end{array}$ \\
\hline$X P-F$ & rare & + & $<25 \%$ & $<25 \%$ & $+1-$ & - & - & $\begin{array}{l}\mathrm{ERCC} 1+ \\
\mathrm{ERCC} 4\end{array}$ & \\
\hline XP-G & rare & ++ & $<10 \%$ & $<10 \%$ & + & ++ & - & ERCC5 & 2 malades atteints de CS \\
\hline$X P$ variant & élevée & + & $100 \%$ & $100 \%$ & + & - & - & $?$ & $\begin{array}{l}\text { Défaut de réparation par } \\
\text { recombinaison }\end{array}$ \\
\hline $\begin{array}{l}\text { Syndrome } \\
\text { de Cockayne } \\
\text { CS-A }\end{array}$ & rare & + & $95 \%$ & $<5 \%$ & - & t+ & - & $?$ & Défaut dans les répara- \\
\hline CS-B & élevée & ++ & $95 \%$ & $<5 \%$ & - & ++ & - & ERCC6 & $\begin{array}{l}\text { trits } \\
\text { crits genes trans- }\end{array}$ \\
\hline CS-C & \multicolumn{8}{|c|}{ identique au XP-B } & Identique au XP-B \\
\hline $\begin{array}{l}\text { Trichothio- } \\
\text { dystrophie } \\
\text { TTD-1 }\end{array}$ & élevée & - & $100 \%$ & $100 \%$ & - & - & + & $?$ & $\begin{array}{l}\text { Pas d'anomalie détecta- } \\
\text { ble de la réparation }\end{array}$ \\
\hline TTD-2 & élevée & + & $<30 \%$ & $?$ & - & + & + & ERCC2 & $\begin{array}{l}\text { Même groupe de com- } \\
\text { plémentation que les } \\
\text { XP-D }\end{array}$ \\
\hline TTD-3 & rare & + & $<20 \%$ & $?$ & - & + & + & $?$ & \\
\hline TTD-4 & rare & + & $<20 \%$ & $?$ & - & + & + & $?$ & \\
\hline
\end{tabular}

ont été caractérisés pour les XP classiques indiquant qu'au moins sept gènes sont capables, indépendamment, de provoquer, après mutation, cette maladie. Le groupe génétiquement différent appelé "XP variant" correspond à des malades moins sensibles aux rayons ultraviolets et déficients dans un autre système de réparation, la réparation par recom$m / s n^{\circ} 1$ vol. 10, janvier 94 binaison ou postréplicative [1]. Aucune donnée génétique n'existe sur ces malades variants.

\section{Le syndrome de Cockayne} et la trichothiodystrophie

Le syndrome de Cockayne (CS) et la trichothiodystrophie (TTD) sont deux autres maladies dans lesquelles une photosensibilité est associée à une anomalie de réparation de l'ADN par excision. Paradoxalement, dans le cas de ces deux maladies, ce défaut de réparation de l'ADN n'est pas associé à un taux de cancer anormal, ni aux anomalies cutanées caractéristiques des malades atteints de Xeroderma pigmentosum dès l'âge de 2-3 ans $[6,7]$. Le syndrome de Cocayne est, en plus de la photo- 


\section{RÉFÉRENCES}

12. Sarasin A, Blanchet-Bardon C, Renault (;) Lehmann A, Arlett C, Dumez Y. Prenatal diagnosis in a subset of trichothiodystrophy patients defective in DNA repair. $\mathrm{Br}$ J Iermatol 1992; 127 : 485-91.

13. Stefanini M, Lagomarsini P, Arlett CF, Borrone C, Crovato F. Trevisan (;, Cordone G, Nuzzo F. Xeroderma pigmentosum (complementation group D) mutation is present in patients affected by trichothiodystrophy with photosensitivity. Hum Genel 1986; 74: 107-12.

14. Eveno E, Chevalier-Lagente O, Benoit A, Carreau M, Vermeulen W, Hoeijmakers JHJ, Stefanini M, Lehmann AR, Weber CA, Mezzina M, Sarasin A. Correction by the ERCC2 gene of UV-sensitivity and repair deficiency phenotype in a subset of trichothiodystrophy cells. Mol Cell Biol 1994, (sous presse).

15. Tanaka K, Miura N, Satokata I, Miyamoto I, Yoshida MC, Satoh Y, Kondo S, Yasui A, Okayama H, Okada Y. Analysis of a human DNA excision repair gene involved in group A Xeroderma pigmentosum and containing a zinc-finger domain. Nalure 1990 ; 348: 73-6.

16. Moustacchi E, Diatloff-Zito C. L'anémie de Fanconi : aspects génétiques et moléculaires. médecine/sciences 1987 ; 3 : 608-12.

17. Weber CA, Salazar EP, Stewart SA, Thompson LH. ERCC-2: cDNA cloning and molecular characterization of a human nucleotide excision repair gene with high homology to yeast RAD3. EMBO / 1990 ; 9 : 1437-47.

18. Weeda G, van Ham RCA, Vermeulen W, Bootsma D, van der Eb AJ, Hoeijmakers JHJ. A presumed DNA helicase encoded by ERCC-3 is involved in the human repair disorders Xeroderma pigmentosum and Cockayne's syndrome. Cell 1990; 62 : 777-91.

19. Mudgett JS, MacInnes MA. Isolation of the functional human excision repair gene ERCC5 by intercosmide recombination. Genomics 1990 ; 8 : 623-33.

20. Troelstra C, van Gool A, de Wit J, Vermeulen W, Bootsma D, Hoeijmakers JHJ. ERCC6, a member of a subfamily of putative helicases, is involved in Cockayne's syndrome and preferential repair of active genes. Cell 1992; 71 : 939-53.

21. James MR, Sarasin A, Perricaudet M, Joab I. Constitutive and inducible expression of Epstein-Barr virus nuclear antigen 3 carried on stable episomal vectors in human cells. Gene 1990 ; 86 : 233-9.

22. Legerski R, Peterson C. Expression cloning of a human DNA repair gene involved in Xeroderma pigmentosum group C. Nalure 1992; 359 : 70-3.
Cockayne est, en plus de la photosensibilité, caractérisé par un nanisme, des anomalies de la rétine et du squelette, une microcéphalie et des dégénérescences neurologiques progressives. Sur le plan génétique, au moins trois groupes de complémentation ont été définis et le défaut biochimique se situe au niveau de la réparation des gènes activement transcrits [8]. Cette voie de réparation, découverte pour la première fois dans les cellules de mammifères puis confirmée dans la levure et chez E. coli, a pour rôle de lever l'inhibition de la synthèse d'ARN bloquée par les lésions sur l'ADN (figure 1). Le maintien d'une synthèse d'ARN à un niveau suffisant est vital pour la cellule qui a besoin, pour une activité cellulaire normale, de l'expression de ses gènes activement transcrits. En effet, dans toute cellule humaine normale traitée par un agent génotoxique, la réparation de l'ADN s'effectue rapidement sur le brin transcrit des gènes actifs, puis sur le brin complé-

mentaire non transcrit, suivie quelques heures plus tard par une réparation généralisée de $95 \%$ de l'ADN cellulaire non actif $[9,10]$. Les cellules CS sont capables de réparer ces $95 \%$ de l'ADN cellulaire (la quantification globale de la réparation dans ces cellules est donc identique à celle obtenue dans les cellules normales), mais ne peuvent réparer les gènes transcrits (c'est-àdire l'ADN fortement lié à la matrice nucléaire) [8]. Ce défaut se traduit par une incapacité de récupérer une synthèse d'ARN normale après irradiation par les rayons ultraviolets et par une hypersensibilité cellulaire aux rayons ultraviolets similaire à celle observée pour les cellules XP. L'image inverse du syndrome de Cockayne est trouvée parmi les malades atteints de Xeroderma pigmentosum appartenant au groupe de complémentation C. En effet, les cellules XP-C sont incapables de réparer l'ensemble de leur ADN génomique (défaut $>90 \%$ ), mais réparent parfaitement bien

Figure 1. Schéma du mécanisme de la réparation de I'ADN par excision-resynthèse dans les cellules humaines. L'ensemble de l'ADN est réparé suivant le schéma de droite ("chromatine inactive/réparation globale ") tandis que I'ADN activement transcrit à partir d'un promoteur $P$ ("chromatine active/réparation préférentielle") est réparé de façon préférentielle et rapide. Réparation globale: (I) la distorsion locale de l'hélice d'ADN par une lésion non codante (rectangle rouge) est reconnue par un complexe multi-enzymatique comprenant au moins les produits des gènes XPAC, XPCC et celui impliqué dans le XPE (EXPEC). (II) Les deux brins de I'ADN endommagé sont ensuite séparés par l'action de deux ADN hélicases (les produits des gènes ERCC2 et ERCC3). (III) L'ensemble de ces protéines, y compris peut-être d'autres protéines non encore identifiées (dont ERCC1, ERCC4 et ERCC5), forme un complexe de grande taille dont l'activité endonucléasique clive le brin d'ADN endommagé, 22-24 nucléotides en $5^{\prime}$ de la lésion et 4-6 nucléotides en 3'. (IV) L'ADN simple brin ainsi formé est immédiatement recouvert de protéines (HSSB: human single-stranded binding protein) afin d'être protégé d'éventuelles dégradations. La brèche formée par l'excision de l'oligonucléotide contenant la lésion est comblée par la synthèse d'ADN initiée en présence de protéines spécifiques (RF-C, PCNA) par I'ADN polymérase $\delta$ ou $\varepsilon$. (V) Le brin nouvellement synthétisé est ligaturé à l'ADN parental grâce à une ADN ligase. Réparation préférentielle. (VI) L'initiation de la transcription au niveau du promoteur $P$ d'un gène activement transcrit par l'ARN polymérase II nécessite de nombreuses protéines, formant le complexe basal de transcription, ainsi que l'activité hélicase du produit du gène ERCC3. (VII) L'ARN polymérase bloquée par une lésion est reconnue par les produits des gènes ERCC6, ERCC3 et peut-être ERCC2. (VIII) L'activité ARN hélicase de ces trois enzymes permet de déplacer l'ARN polymérase de son substrat ainsi que l'ARNm tronqué en cours de synthèse. Les autres enzymes de réparation de I'ADN interviennent alors de façon à créer le complexe d'excision identique à celui utilisé dans la voie de réparation globale (III). (IX) La réparation préférentielle de I'ADN, déficiente dans le syndrome de Cockayne, est la seule à fonctionner chez les malades atteints de Xeroderma pigmentosum groupe $C(X P-C)$, qui ne sont capables que de réparer les lésions sur le brin transcrit des gènes actifs. 


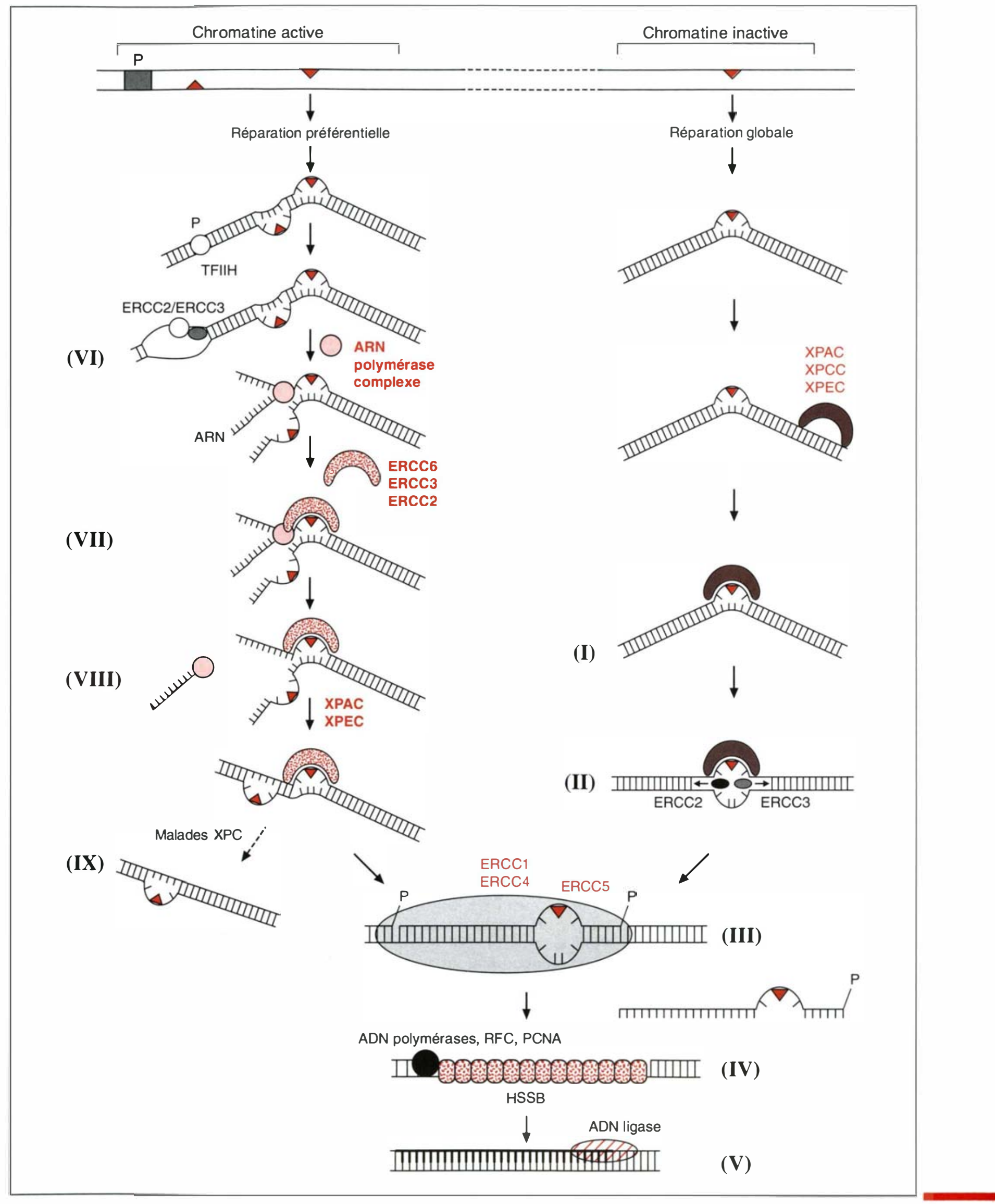




\section{RÉFÉRENCES}

23. Chu G, Chang E. Xeroderma pigmentosum group E cells lack a nuclear factor that binds to damaged DNA. Science 1988 242: $564-7$.

24. Wood RI), Coverley D. DNA excision repair in mammalian cell extracts. Bio Essays 1991; 13: 447-53.

25. 0'Donnovan A, Wood RD. Identical defects in DNA repair in Xeroderma pigmentosum group $G$ and rodent ERCC. group 5. Nalure 1993; 363 : 185-8.

26. Shivji MKK, Kenny MK, Wood RD. Proliferating cell nuclear antigen is required for DNA excision repair. Cell 1992 ; 69 : $367-74$

27. Satokata l, Tanaka K, Miura N, Miyamoto I, Satoh Y, Kondo S, Okada Y. Characterization of a splicing mutation in group A Xeroderma pigmentosum. Proc Nall Acad Sci USA 1990; 87 : 9908-12.

28. Cleaver JE, Cortès F, Lutze LH, Morgan WF, Player AN, Mitchell DL. Unique DNA repair properties of a Xeroderma pigmentosum revertant. Mol Cell Biol 1987 $7: 3353-7$.

29. Kaur GP, Bagga PS, Rinaldy A, Llyod RS, Athwal RS. Two gene hypothesis for DNA repair in XP-A; identification of second gene on human chromosome 8 . Am J Hum Genet 1991 ; 49S : 409.

30. Van Duin M, de Wit J, Odijk H, Westerveld A, Yasui A, Koken MHM, Hoeijmakers JHJ, Bootsma D. Molecular caracterization of the human excision repair gene ERCC-1 :cDNA cloning and amino acid homology with the yeast DNA repair gene RAD10. Cell 1986; 44 : 913-23.

31. Biggerstaff M, Szymkowski DE, Wood RI). Co-correction of the ERCCII, ERCC.4 and Xeroderma pigmentosum group $\mathrm{F}$ DNA repair defects in vitro. EMBO J 1993 ; 12 : 3685-92.

32. Van Vuuren AJ, Appeldoom E, Odijk $H$, Yasui A, Jaspers NGS, Bootsma D, Hoeijmakers JHJ. Evidence for a repair enzyme complex involving ERCCI and complementing activities of ERCC4, ERCCII and Xeroderma pigmentosum
l'ADN des gènes transcrits (figure 1). Cela se traduit par une hypersensibilité cellulaire aux rayons ultraviolets plus faible que pour les cellules des autres malades atteints de Xeroderma pigmentosum, bien que le taux de réparation globale de l'ADN soit quantitativement très faible chez le malade atteint de Xeroderma pigmentosum du groupe C [11]. Un très faible nombre de malades atteints du syndrome de Cockayne (une dizaine actuellement) présentent un tableau clinique remarquable puisqu'il associe en même temps les symptômes typiques du syndrome de Cockayne et ceux du Xeroderma pigmentosum. Ces malades sont génétiquement dispersés parmi plusieurs groupes de complémentation XP (groupes B, D et G), ce qui indique que ces deux maladies dans leur état extrême peuvent être liées et qu'éventuellement des défauts enzymatiques de réparation pourraient être communs à ces deux syndromes rares $[6,7]$.

La trichothiodystrophie est cliniquement caractérisée par un taux faible d'acides aminés soufrés et des anomalies des phanères, en particulier des cheveux cassants et très fins dont la structure microscopique est formée d'une alternance de bandes claires et foncées caractéristique de cette maladie [ $6,7,12]$. Parmi ces malades, environ $50 \%$ sont photosensibles et leurs cellules ont un défaut de réparation de l'ADN. Actuellement, quatre groupes génétiquement différents ont été déterminés, ce qui démontre à nouveau une hétérogénéité génétique importante, comme c'est le cas pour les malades atteints de Xeroderma pigmentosum et de syndrome de Cockayne. Parmi les malades photosensibles, environ $90 \%$ d'entre eux sont génétiquement identiques aux malades atteints de Xeroderma pigmentosum du groupe D[13]. De fait, nous avons récemment démontré que le gène muté dans le Xeroderma pigmentosum groupe D (le gène ERCC2, voir ci-dessous) était aussi capable de permettre une récupération totale des fonctions cellulaires TTD après irradiation par les rayons ultraviolets [14]. Bien que ce même gène ERCC2 soit muté dans ces deux maladies, les manifes- tations cliniques sont paradoxalement complètement différentes puisque les malades atteints de trichothiodystrophie ne développent aucune tumeur de la peau, ni aucune anomalie majeure de la peau exposée au soleil, comme c'est le cas des malades atteints de Xeroderma pigmentosum. Ce résultat conduit à s'interroger sur le rôle intrinsèque de la protéine ERCC2 dans le processus de cancérisation.

\section{Stratégies de clonage des gènes de réparation}

Au moins douze groupes de complémentation différents, correspondant à des anomalies de la réparation de l'ADN, ont été décrits chez l'homme (Tableau I). Ce chiffre est à rapprocher des quatre groupes isolés chez $E$. coli et d'au moins dix groupes chez la levure. On devrait donc s'attendre au clonage et à la caractérisation d'une douzaine de gènes de réparation chez l'homme. Plusieurs stratégies de clonage ont été utilisées.

Transfert d'ADN génomique humain nornal dans les cellules humaines déficientes en réparation

La stratégie théoriquement la plus simple et la plus évidente a consisté à transfecter de l'ADN génomique isolé de cellule normale dans les cellules en culture provenant de malades et de sélectionner des révertants pour une meilleure résistance aux lésions par les rayons ultraviolets. Le gène de réparation est alors isolé par les méthodes classiques de la biologie moléculaire. Cette technique s'est révélée d'application difficile avec des cellules humaines, en particulier du fait de leur faible capacité à intégrer de façon stable de l'ADN de haut poids moléculaire, d'origine exogène. Le seul succès de cette technique a été l'isolement du gène capable de complémenter le Xeroderma pigmentosum, groupe A [15]. Ce succès a été essentiellement dû à la ténacité des auteurs japonais (il fallait commencer l'expérience avec plus de $10^{9}$ fibroblastes XP en culture) et aussi à la très grande sensibilité des cellules XP-A aux rayons ultraviolets, et donc à une différence énorme de sélectivité 
entre cellules sensibles et cellules résistantes aux rayons ultraviolets.

Transfert d'ADN génomique humain normal dans des cellules de rongeurs déficientes en réparation de l'ADN

La difficulté technique d'utiliser directement les cellules de malades comme cellule hôte pour transfecter l'ADN, et l'intérêt d'isoler des gènes de réparation dont le dysfonctionnement serait incompatible avec un développement embryonnaire normal, ont conduit plusieurs équipes à fabriquer in vitro des cellules de rongeurs déficientes en réparation de l'ADN. En effet, il n'existe pas de modèles animaux de ces maladies humaines d'hypersensibilité aux rayons ultraviolets. Une mutagenèse généralisée de cellules de hamster (lignées CHO et V79) ou de souris (lignée L5178Y) a permis d'isoler de nombreuses lignées sensibles aux rayons ultraviolets qui ont été classées en douze groupes de complémentation. Parmi ces cellules, quelques lignées sont aussi particulièrement sensibles aux agents pontant les deux brins de l'ADN (mitomycine $\mathrm{C}$, psoralène + rayons ultraviolets $\mathrm{A})$ et représentent un modèle pour cette autre maladie de la réparation qu'est l'anémie de Fanconi $[1,16]$.

La grande facilité avec laquelle les cellules de rongeurs sont capables d'accepter et d'intégrer de l'ADN génomique exogène a permis d'isoler des gènes humains capables de complémenter in vitro la sensibilité aux rayons ultraviolets de ces cellules de rongeurs déficientes en réparation. Ces gènes ont été appelés ERCC (excision repair cross complernenting). Cinq gènes $\operatorname{ERCC~}(1,2,3,5$ et 6) ont été isolés grâce à leur capacité de complémenter les groupes de complémentation 1, 2, 3, 5 et 6 des cellules $\mathrm{CHO}$ sensibles aux rayons ultraviolets. Parmi ces gènes, trois sont impliqués dans le Xeroderma pigmentosum (ERCC2, 3 et 5) $[17,19]$ et un dans le syndrome de Cockayne (ERCC6) [20].

Transfert de banque d'ADNc dans les cellules humaines déficientes en réparation de l'ADN

Le développement de vecteurs $m / s n^{\circ} 1$ vol. 10 , janvier 94 d'expression extrachromosomiques fondés sur l'origine de réplication $\mathrm{du}$ virus $\mathrm{EBV}[21,22]$ a permis d'utiliser des banques d'ADNc pour complémenter directement les cellules de malades sensibles aux rayons ultraviolets. Cette stratégie a été couronnée de succès pour l'isolement du gène humain déficient dans le Xeroderma pigmentosum de groupe $\mathrm{C}$ (gène XPCC) [22]. Le maintien de ces vecteurs sous forme épisomique avec un nombre relativement important de copies par cellule permet d'éviter les écueils liés à l'utilisation d'ADN génomique de haut poids moléculaire. De plus, le vecteur épisomique est facilement récupérable à partir de la cellule hôte rendue résistante aux rayons ultraviolets et le gène est déjà cloné dans un vecteur navette. Cette stratégie représente actuellement la technique de choix pour l'isolement de gènes de réparation.

Micro-injection d'ARNm ou de protéines purifiées dans des cellules humaines déficientes en réparation de I'ADN

I a complémentation d'un défaut de réparation peut être obtenue par la micro-injection d'ARNm ou de protéines purifiées de cellules normales. Le fractionnement d'extraits cellulaires normaux en fonction de la taille du message ou des caractéristiques biochimiques attendues d'une protéine permet en principe de purifier, puis d'isoler le facteur déficient. L'obtention d'ADNc à partir de la protéine purifiée est ensuite envisageable. Ce protocole est très lourd et n'a pas permis jusqu'à maintenant d'isoler un gène de réparation. Une protéine de $120 \mathrm{kDa}$, ayant une affinité importante pour l'ADN irradié aux rayons ultraviolets ou endommagé par le cis-platine, a été partiellement purifiée à partir de cellules normales et on a montré qu'elle faisait défaut dans le Xeroderma pigmentosum de groupe $\mathrm{E}$ [23]. Le clonage de l'ADNc est actuellement en cours.

Complémentation d'extraits acellulaires déficients par des protéines purifiées normales, dans un test de réparation in vitro

Des extraits acellulaires provenant de cellules normales sont capables d'effectuer in vitro la réparation de l'ADN d'un plasmide endommagé par des agents génotoxiques [24]. Des extraits isolés de cellules XP ne permettent pas cette réparation et peuvent être complémentés par l'addition de fractions plus ou moins purifiées de cellules normales. Ce test de complémentation permet en principe d'isoler des protéines de réparation et éventuellement de cloner les gènes correspondants. De fait, tout à fait récemment, on a montré que la fraction protéique capable de complémenter dans ce test le XP-G était identique à la protéine déficiente dans le groupe 5 des cellules $\mathrm{CHO}$ sensibles aux rayons ultraviolets. Ce résultat confirme que le gène ERCC5 est responsable du Xeroderma pigmentosum de groupe G [25]. Un des intérêts supplémentaires de cette technique consiste à déterminer les protéines nécessaires à la réparation mais pour lesquelles il n'existe pas de mutants, probablement du fait de leur non-viabilité. Il faut rappeler que parmi les nombreux gènes codant pour des enzymes impliquées dans les mécanismes du maintien de l'information génétique, au sens large du terme, seuls "les mutants" de la réparation de l'ADN sont connus chez l'homme. Les essais in vitro ont permis de montrer que les étapes de réparation de la brèche créée après l'excision de l'oligonucléotide (28-30 mers dans les cellules de mammifères, voir figure 1) nécessitaient une protéine de liaison à l'ADN (HSSB : human single-strand binding protein ou RF-A), les ADN polymérases $\delta$ ou $\varepsilon$, ainsi que le PCNA (proliferating cell nuclear antigen) et le RF-C, protéines nécessaires à la réplication du génome du virus SV40[26]. Ces protéines auraient pour rôle de compléter la brèche après excision de l'oligonucléotide modifié et de répliquer un petit morceau d'ADN en utilisant le brin opposé comme matrice (figure 1). Ces mêmes enzymes sont aussi impliquées dans la réplication semi-conservative normale. On peut donc facilement imaginer que des mutations sur ces protéines soient létales. La complémentarité de ces études in vitro avec l'isolement

des 


\section{RÉFÉRENCES}

33. Gulyas KD, Donahue TF. SSI.2, a suppressor of a stem-loop mutation in the HIS4 leader encodes the yeast homolog of human ERCC-3. Cell 1992; 69: 1031-42.

34. Mounkes LC, Jones RS, Liang BC, Gelbart W, Fuller MT. A drosophila model for Xeroderma pigmentosum and Cockayne's syndrome : haywire encodes the fly homolog of ERCC3, a human repair gene. Cell 1992 ; 71 : 925-37.

35. Schaeffer L, Roy R, Humbert S, Moncollin V, Vermeulen W, Hoeijmakers JH J, Chambon P, Egly JM. DNA repair helicase : a component of BTF2 (TFIIH) basic transcription factor. Science 1993; 260 : 58-63.

36. Friedberg EC. Xeroderma pigmentosum, Cockayne's syndrome, helicases, and DNA repair: what's the relationship? Cell 1992, 71: 887-9.

37. Selby CP, Sancar A. Molecular mechanism of transcription-repair coupling. Science 1993 ; 260 : 53-8.

38. Vuillaume $\mathrm{M}$, Calvayrac $\mathrm{R}$, BestBelpomme M, Tarroux P, Hubert M, Decroix R, Sarasin A. Deficiency in the catalase activity of Xeroderma pigmento sum cell and simian virus 40-transformed human cell extracts. Cancer Res 1986 ; 46 : 538-44.

39. Madzak C, Armier J, Stary A, DayaGrosjean L, Sarasin A. UV-induced mutations in a shuttle vector replicated in repair deficient trichothiodystrophy cells differs with those in genetically-related cancer prone Xeroderma pigmentosum. Carcinogenesis 1993; 14 : 1255-60.

40. Daya-Grosjean L, Robert C, Drougard C, Suarez HG, Sarasin A. High mutation frequency in ras genes of skin tumors isolated from DNA-repair deficient Xeroderma pigmentosum patients. Cancer Res 1993 ; 53 : 1625-9.

41. Dumaz N, Drougard C, Sarasin A, Daya-Grosjean L. Specific UV-induced mutation spectrum in the p53 gene of skin tumors from DNA repair deficient Xeroderma pigmentosum patients. Proc Natl Acad Sci USA 1993; 90 : 10529-33.

42. Vuillaume M, Daya-Grosjean L, Vincens P, Pennetier JL, Tarroux P, Baret A, Calvayrac R, Sarasin A. Striking differences in cellular catalase activity between two DNA repair-deficient diseases : Xeroderma pigmentosum and trichothiodystrophy. Car cinogenesis 1992; 13: 321-8. gènes de réparation est nécessaire pour analyser en détail toutes les étapes de la réparation chez l'homme.

\section{Structure et rôle des gènes de réparation}

Grâce à l'utilisation des stratégies de clonage décrites ci-dessus, six des gènes de réparation impliqués dans des maladies humaines d'hypersensibilité aux rayons ultraviolets ont été isolés et caractérisés (Tableau II). Le gène XPAC (pour XP-A correcting factor) est capable de corriger la sensibilité aux rayons ultraviolets des cellules isolées de malades atteints de Xeroderma pigmentosum de groupe A. Les caractéristiques de ce gène sont indiquées sur le Tableau II. Le gène code pour une protéine de poids moléculaire apparent d'environ $40 \mathrm{kDa}$; d'après la séquence, elle doit posséder une structure en doigt de zinc et une activité de liaison à l'ADN [15]. L'affinité de la protéine pour l'ADN irradié est 1000 fois plus élevée que pour l'ADN natif. Le gène RADl14 de levure a une grande homologie de structure avec le gène XPAC; les mutants rad 14 sont totalement dépourvus d'activité de coupure du brin d'ADN endommagé par les rayons ultraviolets. Les patients XP$A$, qui ont un grave défaut de réparation de l'ADN ( $<5 \%$ du témoin), présentent un phénotype clinique très sévère avec un nombre important d'anomalies neurologiques. C'est dans ce groupe de complémentation que les premières mutations sur un gène humain de réparation ont été identifiées [27]. Ce groupe est très important au Japon où $80 \%$ des malades ont la même mutation ponctuelle $(G \rightarrow C)$ sur le site accepteur d'épissage en 3' de l'intron 3 sur au moins un des allèles de ce gène, indiquant clairement l'existence d'une néo-mutation unique chez un ancêtre des malades japonais actuels atteints de Xeroderma pigmentosum [25]. Cette mutation entraîne une instabilité de l'ARNm et la synthèse d'une protéine tronquée inactive. Le rôle primordial de la protéine XPAC dans la reconnaissance des lésions induites par les rayons ultraviolets est attesté par l'existence d'une lignée de cellules XPA qui n'est plus sensible aux rayons ultraviolets après mutation ponctuelle sur le site muté originellement; l'acide aminé responsable du phénotype muté est remplacé par un autre acide aminé différent de celui présent dans la protéine sauvage. Le phénotype de cette nouvelle protéine n'est que partiellement normal car la cellule correspondante ne répare qu'une partie des lésions induites par les rayons ultraviolets, les pyrimidine (6-4)pyrimidones, mais reste déficiente dans la réparation des dimères de pyrimidines [28]. La protéine XPAC doit donc être directement impliquée dans la reconnaissance de la lésion ou de la distorsion structurale produite par celleci (figure 1). Un autre gène, différent de XPAC (il serait sur le chromosome 8 ), permet une réversion partielle du phénotype XPA; il a été isolé, mais son rôle exact dans la réparation de l'ADN est encore inconnu [29].

Le gène XPCC complémentant le déficit des cellules XP-C a été récemment isolé. Le produit de ce gène doit intervenir dans la réparation de la chromatine inactive, puisque les cellules XP-C sont capables de réparer les gènes activement transcrits mais sont complètement incapables de réparer le reste de l'ADN. La structure du gène XPCC suggère pour la protéine qui en est issue un site de liaison à l'ADN et une activité ATPasique dépendante de l'ADN [22].

L'utilisation des cellules de rongeurs a permis l'isolement de six gènes de réparation (ERCCl, 2, 3, 4, 5 et 6 ) dont quatre au moins sont liés à des maladies humaines. Le gène ERCCl n'a pas pu être lié à une maladie connue. Sa structure comporte une certaine homologie avec le gène de réparation de la levure RAD10, qui intervient dans des processus de recombinaison de l'ADN [30]. Tout à fait récemment, une association forte a été démontrée entre les produits des gènes ERCCl et ERCC4, analogue à celle qui existe entre les protéines de levure RADl0 et RAD1 [31, 32]. Ce dernier complexe aurait une activité endonucléasique nécessaire à la fois aux processus 
Tableau II

CARACTÉRISTIQUES DES GĖNES ET DES PROTÉINES HUMAINS IMPLIQUÉS DANS LA RÉPARATION DE L'ADN

\begin{tabular}{|c|c|c|c|c|c|c|c|}
\hline Gène & $\begin{array}{c}\text { Localisation } \\
\text { chromosomique }\end{array}$ & $\begin{array}{l}\text { Taille } \\
\text { du gène } \\
\text { (kB) }\end{array}$ & $\begin{array}{l}\text { Taille de } \\
\text { la protéine } \\
\text { (acides } \\
\text { aminés) }\end{array}$ & $\begin{array}{l}\text { Homologie } \\
\text { avec } \\
\text { d'autres } \\
\text { gènes* }\end{array}$ & $\begin{array}{l}\text { Rôle dans } \\
\text { une maladie } \\
\text { humaine }\end{array}$ & $\begin{array}{l}\text { Caractéristiques } \\
\text { supposées } \\
\text { de la protéine }\end{array}$ & Références \\
\hline ERCC1 & $19 q 13.2$ & 15 & 297 & $\begin{array}{l}\text { uvrA/uvrC** } \\
\text { RAD } 10\end{array}$ & \multirow{2}{*}{$X P-F$} & $\begin{array}{l}\text { - Site de liaison à } \\
\text { I'ADN }\end{array}$ & {$[30]$} \\
\hline ERCC4 & $16 p$ & $?$ & $?$ & RAD 1 (?) & & $\begin{array}{l}\text { - Impliquée dans la } \\
\text { recombinaison? }\end{array}$ & {$[31,32]$} \\
\hline ERCC2 & $19 q 13.2$ & $\cong 20$ & 760 & RAD3 & XP-D/TTD-2 & $\begin{array}{l}\text { - ADN/ARN hélicase } \\
5^{\prime} \rightarrow 3^{\prime}\end{array}$ & [17] \\
\hline ERCC3 & $2 q 21$ & $\cong 45$ & 782 & $\begin{array}{l}\text { SSL2/RAD25 } \\
\text { haywire*** }\end{array}$ & XP-B/CS-C & $\begin{array}{l}\text { - ADN/ARN hélicase } \\
3^{\prime} \rightarrow 5^{\prime} \text { dépendante } \\
\text { de I'ATP } \\
\text { - Composant du fac- } \\
\text { teur de transcri- } \\
\text { ption TFIIH }\end{array}$ & [18] \\
\hline ERCC5 & 13q32.3-33. 1 & $\cong 32$ & 1186 & RAD2 & XP-G & $\begin{array}{l}\text { - Endonucléase } \\
\text { spécifique pour } \\
\text { I'ADN simple brin }\end{array}$ & [19] \\
\hline ERCC6 & $10 q 11-21$ & $\cong 85$ & 1493 & $?$ & CS-B & $\begin{array}{l}\text { - ADN/ARN hélicase } \\
\text { impliquée dans la } \\
\text { réparation des gè- } \\
\text { nes activement } \\
\text { transcrits }\end{array}$ & [20] \\
\hline XPAC & $9 q 34.1$ & $\cong 25$ & 273 & RAD 14 & $X P-A$ & $\begin{array}{l}\text { - Site de liaison à } \\
\text { I'ADN simple et } \\
\text { double brin (en- } \\
\text { dommagé par les } \\
\text { UV) } \\
\text { - protéine à doigt de } \\
\text { zinc }\end{array}$ & [15] \\
\hline XPCC & $?$ & $?$ & 823 & RAD 4 & $X P-C$ & $\begin{array}{l}\text { - Site de liaison à } \\
\text { l'ADN et à la chro- } \\
\text { matine } \\
\text { - ATPase dépen- } \\
\text { dante de I'ADN }\end{array}$ & [22] \\
\hline
\end{tabular}

(*) Gènes isolés chez la levure sauf $\left(^{* *}\right)$ isolé chez E. coli et (**) isolé chez la drosophile.

de réparation des dommages de l'ADN, mais aussi à la recombinaison mitotique. Il semble que le gène ERCC4, ou que les deux gènes ERCCl et ERCC4, soient impliqués dans le Xeroderma pigmentosum de groupe F [29, 30].

Le gène ERCC2 qui code pour une protéine de 760 acides aminés a une structure comportant sept domaines caractéristiques de la famille des hélicases [17]. Le gène ERCC2 a $50 \%$ d'homologie avec le gène de $\mathrm{m} / \mathrm{s} \mathrm{n}^{\circ} 1 \mathrm{vol} .10$, janvier 94 levure RAD3 dont le produit a, in vitro, une activité $\mathrm{ADN} / \mathrm{ADN}$ et ADN/ARN hélicase à polarité 5'-3' dépendante de l'ATP. Ce gène, dont le produit est absolument nécessaire à la réparation par excision, est vital chez la levure et son inactivation totale entraîne la mort du mutant. On pense que l'activité hélicase reconnaît la présence d'une lésion changeant la structure secondaire de l'ADN et permet l'incision du brin endommagé en séparant les deux brins de l'ADN. Un autre rôle possible de la protéine ERCC2 pourrait être de déplacer, à la fin de la réparation, l'oligonucléotide portant la lésion afin de permettre un recyclage des enzymes de réparation. Cette activité spécifique de l'hélicase a été trouvée chez les bactéries (gène $u v r D$ ) et est présumée pour le produit du gène RAD3 chez la levure. Le gène ERCC2 normal est capable de complémenter deux classes de cellules différentes: les cellu- 
les de malades appartenant au groupe I) de Xeroderma pigmentosum et celles de la plupart des malades photosensibles atteints de trichothiodystrophie. Dans ces deux cas, le gène ERCC2 sauvage complémente la sensibilité cellulaire aux rayons ultraviolets ainsi que le défaut de réparation des lésions induites par les rayons ultraviolets [14]. Nous ne savons pas encore si les domaines fonctionnels mutés de ERCC2 sont les mêmes dans les deux syndromes (figure 2).

Le gène ERCC3, capable de complémenter le groupe de complémentation 3 des cellules $\mathrm{CHO}$, est impliqué dans le Xeroderma pigmentosum groupe B [18]. Le petit nombre de malades atteints de Xeroderma pigmentosum groupe B est toujours atteint à la fois de Xeroderma pigmentosum et du syndrome de Cockayne et des mutations affectent leurs gènes ERCC3. Il est donc possible que le produit du gène ERCC3 soit à l'origine de deux maladies présentes chez le même malade. ERCC3 code pour une protéine de 782 acides aminés, comportant un signal de localisation nucléaire et un domaine caractéristique des ADN hélicases. Son analogue dans la levure, le gène RAD25 ou SSL2, est impliqué dans le blocage de la traduction d'ARN messagers contenant des structures palindromiques. Ce rôle de régulation pourrait être lié à l'activité ARN hélicase dépendante de l'ATP de l'enzyme capable d'éliminer les structures secondaires impliquées dans le contrôle de l'initiation de la synthèse protéique [33]. Chez la levure, ce gène a une fonction vitale, indépendante de la réparation de l'ADN, ce qui explique peut-être le faible nombre de malades atteints de Xeroderma pigmentosum groupe $\mathrm{B}$, si cette fonction vitale est conservée chez l'homme (figure 2). Le gène humain ERCC3 possède une grande homologie avec le gène haywire de drosophile [34], dont le rôle exact n'est pas encore connu. Toutefois, les mutations sur ce gène de drosophile, lorsqu'elles ne sont pas létales, s'expriment par un phénotype de sensibilité aux rayons ultraviolets, de stérilité et d'anomalies de développement du système nerveux central. Ces carac-

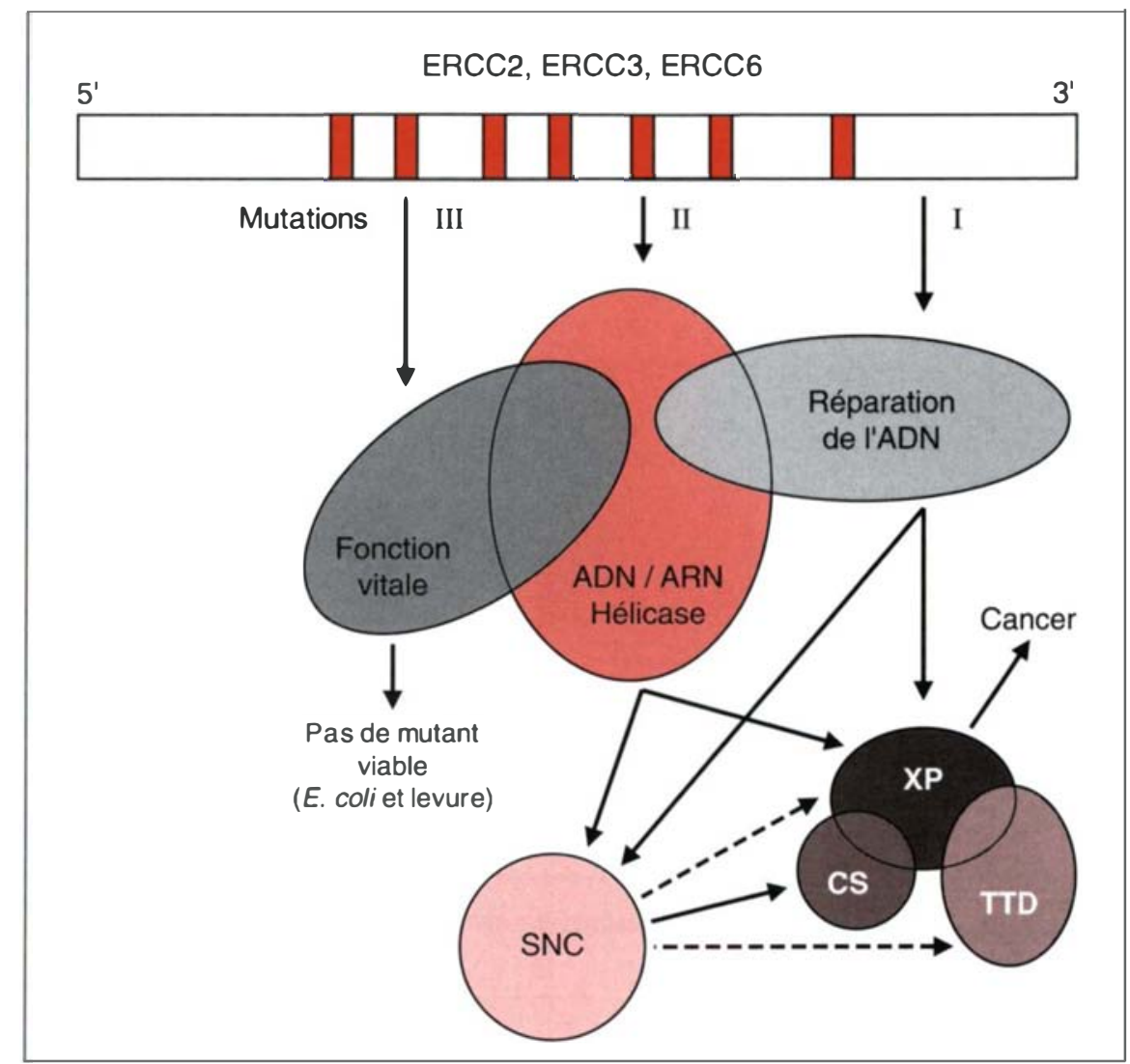

Figure 2. Modèle concernant l'effet pléiotropique des mutations sur les gènes de réparation de I'ADN. Les gènes ERCC2, ERCC 3 et ERCC6 possèdent des séquences caractéristiques des domaines ADN/ARN hélicases (zones rouges). Des mutations localisées dans la partie 3'-terminale du gène donneront naissance à des enzymes faiblement altérées dans le domaine de la réparation de l'ADN (I). Les malades correspondants seront relativement peu atteints. Des mutations dans certains domaines de l'activité hélicase donneront naissance à des enzymes très altérées dans la réparation de l'ADN, probablement à cause d'une anomalie sévère dans l'activité hélicase. Les malades portant cette mutation seront sévèrement déficients au niveau de la réparation de l'ADN et dans le fonctionnement de leur système nerveux central (SNC). Des phénotypes extrêmes ayant à la fois les symptômes de syndrome de Cockayne et de Xeroderma pigmentosum pourraient être dus à ce type de mutation (II). Enfin, des mutations sur les sites vitaux de ces enzymes peuvent être incompatibles avec le développement normal de l'embryon. Cette fonction vitale ne peut être mise en évidence qu'en utilisant des mutants conditionnels chez la levure ou la bactérie (III). 
téristiques sont présentes chez les malades atteints de Xeroderma pigmentosum groupe B. Tout à fait récemment, l'équipe de J.M. Egly a démontré que ERCC3 faisait partie du complexe minimal d'initiation de la transcription (TFIIH) et correspondrait à l'activité hélicase 3'-5' dépendante de l'ATP et de l'ADN, qui sépare les deux brins d'ADN pour débuter la transcription $(\mathrm{m} / \mathrm{s}$ $n^{\circ} 4$, vol. 9, p. 485) [35]. Comme le syndrome de Cockayne est caractérisé par l'impossibilité de réparer l'ADN des gènes en cours de transcription, ces résultats permettent de jeter un pont entre initiation de la transcription et réparation préférentielle des gènes activement transcrits (figure 1).

Le gène ERCC5 a été isolé grâce à sa capacité de complémenter le groupe 5 des cellules $\mathrm{CHO}$ sensibles aux rayons ultraviolets. Ce gène est impliqué dans le Xeroderma pigmentosum groupe $\mathrm{G}$. La fonction du produit de ce gène est encore inconnue [19, 25], mais son homologue chez la levure RAD2 aurait une activité endonucléique sur l'ADN simple brin.

Le gène ERCC6 code pour une des protéines les plus grosses du système de réparation puisqu'elle contient 1493 acides aminés. En dehors d'une activité de liaison à l'ADN et d'un signal de localisation nucléaire, la protéine ERCC6 aurait une activité $\mathrm{ARN}$ et $\mathrm{ADN}$ hélicase comme son homologue de la levure, RAD16. La séquence de 500 acides aminés comportant l'activité potentielle $\mathrm{ADN}$ hélicase est retrouvée dans de nombreuses autres protéines de levure et de drosophile [20]. Ces protéines ont, soit une activité directement liée à la réparation (RADl6, RAD5) ou à la recombinaison (RAD54), soit une activité présumée d'activateur de transcription (SNF2, MOT1, brm) [20, 36]. ERCC6 est le gène impliqué dans le syndrome de Cockayne groupe B (qui regroupe la grande majorité des malades atteints d'un syndrome de Cockayne), responsable de l'incapacité de réparer le brin transcrit des gènes actifs [8].

$m / s n^{\circ} 1$ vol. 10, janvir 94

\section{Rôle des hélicases dans la réparation de I'ADN}

Il peut paraître surprenant que parmi les sept premiers gènes clonés impliqués dans la réparation de l'ADN chez l'homme, trois codent pour une activité hélicase. On peut rappeler ici que chez $E$. coli au moins trois ADN hélicases sont impliquées dans le processus de réparation par excision: le complexe UvrA/UvrB qui se fixe sur la lésion avant l'étape d'incision du brin endommagé, la protéine UvrD qui élimine l'oligonucléotide excisé, et la protéine Mfd. Cette dernière protéine a été mise en cause dans le mutant mfd (mutation frequency decline), incapable d'effectuer la réparation préférentielle du brin transcrit [37]. Ce facteur appelé aussi TRCF (transcription repair coupling factor) pourrait reconnaître spécifiquement l'ARN polymérase bloquée par une lésion, la déplacer ainsi que l'ARNm tronqué, peut-être par son activité ARN hélicase, et faciliter l'accessibilité des enzymes de réparation [37]. Les produits des gènes ERCC3, ERCC6 et peut-être ERCC2 auraient un rôle similaire en reconnaissant et en déplaçant l'ARN polymérase eucaryote de type II bloquée par une lésion (figure 1). Enfin, la nécessité de séparer les deux brins de l'ADN est commune aux voies de réparation de l'ADN et à l'initiation et l'élongation de la transcription et de la réplication. Il n'est donc pas surprenant de retrouver l'hélicase ERCC3 impliquée à la fois dans la réparation et la transcription. On peut imaginer que les autres hélicases de la réparation (ERCC2 et ERCC6) pourraient éventuellement intervenir aussi au niveau de la transcription et/ou de la réplication.

\section{Réparation de I'ADN et système nerveux central}

L'association très étroite de dégénérescence neurologique progressive avec de nombreuses maladies de la réparation de l'ADN (pratiquement toutes les formes de Xeroderma pigmentosum en dehors de la forme C, presque tous les malades atteints d'un syndrome de Cockayne et de nombreux malades atteints de trichothiodystrophie) implique un rôle fondamental des enzymes de réparation pour la survie des neurones. Ces anomalies neurologiques sont variables d'une classe de malades à l'autre, mais correspondent généralement à des retards mentaux progressifs, des réflexes anormaux, des microcéphalies, de l'ataxie. L'implication des enzymes de réparation au niveau des complexes de transcription pourrait se traduire par des anomalies subtiles de l'expression génique expliquant ainsi l'aspect pléiotropique de ces maladies. Chez les malades atteints de Xeroderma pigmentosum (en dehors du groupe XP-C), l'absence de réparation de l'ensemble de l'ADN semble responsable d'une dégradation des axones qui entraîne à son tour une dégénérescence primaire des neurones, tandis que chez les malades atteints d'un syndrome de Cockayne l'absence de réparation des gènes activement transcrits se traduit par une destruction des cellules gliales, suivie par une démyélinisation sévère et progressive du système nerveux central et périphérique. Le défaut de réparation de gènes spécifiques pourrait être à l'origine de ces différents symptômes. L'absence de signes neurologiques majeurs chez les malades atteints de Xeroderma pigmentosum forme $\mathrm{C}$ montre clairement le rôle fondamental de la réparation préférentielle dans le maintien de la survie cellulaire. Il est évident que la destruction des neurones n'est pas due à la présence de lésions induites par les rayons ultraviolets dans leur génome. Il faut donc supposer qu’il existe d'autres lésions produites par des processus endogènes à la cellule nerveuse (produits du métabolisme cellulaire, radicaux libres de l'oxygène) ou provoquées par des substances toxiques environnantes. Les cellules du système nerveux central ont une activité métabolique intense qui pourrait être éventuellement responsable de l'apparition de ces lésions toxiques. Le déficit en catalase des cellules XP est un argument supplémentaire pour invoquer le rôle des espèces activées de l'oxygène dans la destruction neuronale [38]. 


\section{Réparation de I'ADN et cancer}

L'absence de tumeurs cutanées chez. les malades atteints de trichothiodystrophie et de syndrome de Cockayne déficitaires en mécanismes de réparation de l'ADN est encore inexpliquée. $\mathrm{Si}$, dans le cas des cellules CS, l'absence de réparation des gènes activement transcrits, et donc indispensables à une survie cellulaire normale, doit conduire à une mort cellulaire plutôt qu'à la croissance de cellules mutées, le cas des trichothiodystrophies est encore plus compliqué puisque le même gène est impliqué dans cette maladie ainsi que dans le Xeroderma pigmentosum de groupe D. Nous ne savons pas s'il existe des différences significatives parmi les types de mutations trouvées sur les gènes de réparation de ces deux syndromes. Si oui, on peut supposer que des domaines fonctionnels des protéines de réparation sont modifiés différemment et peuvent provoquer des phénotypes cliniques variés (figure 2). Il n'est pas exclu que d'autres protéines soient aussi spécifiquement modifiées chez certains malades [7]. Les cellules de ces trois classes de malades sont hypersensibles et hypermutables après irradiation par les rayons ultraviolets. I,es taux de mutation induits par les rayons ultraviolets dans les cellules XP-D et TTD-2 sont du même ordre de grandeur, c'est-à-dire 10 à 50 fois plus élevés que dans le cas des cellules normales [39]. Cette fréquence de mutation élevée, que l'on retrouve in vivo sur les gènes ras des tumeurs XP [40] n'est associée à un fort taux de cancer que dans les Xeroderma pigmentosum. Le rôle des rayons ultraviolets comme agent initiateur des tumeurs XP est attesté par l'étude du spectre des mutations retrouvées sur le gène p53 suppresseur de tumeurs qui est caractéristique des mutations induites par les rayons ultraviolets [41]. Le spectre mutagène, particulièrement caractéristique des lésions induites par les rayons ultraviolets (transition $\mathrm{C}$ vers $\mathrm{T}$ et double mutation (-C. vers T-T), est retrouvé non seulement sur les oncogènes ras activés et le gène sup- aussi sur les gènes endogènes des cellules de malades traitées in vitro par les ultraviolets. I a concordance des spectres de mutations dans les cellules en culture et dans les tumeurs de malades atteints de Xeroderma pigmentosum est tout à fait impressionnante, et confirme l'importance et le rôle des tests in vitro.

En conclusion, il apparaît évident que les rayons ultraviolets solaires peuvent être considérés comme des agents initiateurs, voire promoteurs, de tumeurs, en particulier chez les malades atteints de Xeroderma pigmentosum, dépourvus de système de réparation par excision. Toutefois, l'induction de mutations induites par les rayons ultraviolets n'est pas suffisante pour produire des tumeurs chez les patients atteints d'un syndrome de Cockayne ou de trichothiodystrophie. (On peut donc supposer que ces malades possèdent des voies biologiques antitumorales déficientes chez. les malades atteints de Xeroderma pigmentosum (la catalase pourrait en partie jouer ce rôle) $[38,42]$, ou que certaines propriétés biochimiques facilitent le développement rapide de tumeurs chez les malades atteints de Xeroderma pigmentosum, telles qu'une activité diminuée de la surveillance immunitaire [6, 7]. I a caractérisation des gènes de réparation et leur utilisation potentielle en thérapie génique permettent d'envisager un avenir plus optimiste pour certains de ces malades

\section{Remerciements}

Je remercie les membres de mon laboratoire qui se sont penchés avec critique et patience sur ce manuscrit et en particulier le Dr A. Stary qui m'a aidé pour l'iconographie.

\section{TIRÉS A PART}

\section{A. Sarasin}

\section{Summary}

The human genes of DNA repair

All living cells must maintain the integrity of their genetic information in order to function normally. The genetic stability is insured by numerous repair pathways which counteract the deleterious consequences of DNA lesions produced by a variety of endogenous or exogenous genotoxics. Among them, UV light is a potent source of hasards in man. The fundamental role of repair pathways is attested by the existence of several human diseases where UV-hypersensitivity due to repair deficiency is associated with cancer-proneness (Xeroderma pigmentosum) or with various other clinical defects (Cockayne's syndrome or trichothiodystrophy). These rare, genetically-transmitted disorders have been used to clone several human DNA repair genes. The study of these genes as well as those involved in DNA repair of drosophila, yeast or bacteria allows us to draw a picture of how excision DNA repair is working in man. A very close relationship between transcription process and preferential repair of active genes has been demonstrated using some of these patients cells. Several of these repair genes code for DNA/RNA helicases which, at least for one, also belong to basal transcription complex. Defects in these genes are also involved in neurological disorders and in the initiation of carcinogenesis. Molecular analysis of the regulation of these genes will give us fondamental information on some diseases and on the possible use of gene therapy. 